\title{
Characterization of smooth muscle cells from human atherosclerotic lesions and their responses to Notch signaling
}

\author{
Jessica Davis-Knowlton $\mathbb{D}^{1,2} \cdot$ Jacqueline E. Turner $^{1} \cdot$ Anna Turner $^{1} \cdot$ Sydney Damian-Loring ${ }^{1} \cdot$ Nicholas Hagler $^{1}$. \\ Terry Henderson ${ }^{1} \cdot$ Ivette F. Emery $^{1} \cdot$ Kyle Bond $^{1} \cdot$ Christine W. Duarte $^{1,2} \cdot$ Calvin P. H. Vary $^{1,2}$. \\ Jens Eldrup-Jorgensen ${ }^{1} \cdot$ Lucy Liaw ${ }^{1,2}$
}

Received: 20 October 2017 / Revised: 24 April 2018 / Accepted: 25 April 2018 / Published online: 23 May 2018

(c) United States \& Canadian Academy of Pathology 2018

\begin{abstract}
Atherosclerosis is the most common cause of heart disease and stroke. The use of animal models has advanced our understanding of the molecular signaling that contributes to atherosclerosis. Further understanding of this degenerative process in humans will require human tissue. Plaque removed during endarterectomy procedures to relieve arterial obstructions is usually discarded, but can be an important source of diseased cells. Resected tissue from carotid and femoral endarterectomy procedures were compared with carotid arteries from donors with no known cardiovascular disease. Vascular smooth muscle cells (SMC) contribute to plaque formation and may determine susceptibility to rupture. Notch signaling is implicated in the progression of atherosclerosis, and plays a receptor-specific regulatory role in SMC. We defined protein localization of Notch2 and Notch3 within medial and plaque SMC using immunostaining, and compared Notch2 and Notch3 levels in total plaques with whole normal arteries using immunoblot. We successfully derived SMC populations from multiple endarterectomy specimens for molecular analysis. To better define the protein signature of diseased SMC, we utilized sequential window acquisition of all theoretical spectra (SWATH) proteomic analysis to compare normal carotid artery SMC with endarterectomy-derived SMC. Similarities in protein profile and differentiation markers validated the SMC identity of our explants. We identified a subset of differentially expressed proteins that are candidates as functional markers of diseased SMC. To understand how Notch signaling may affect diseased SMC, we performed Jagged1 stimulation of primary cultures. In populations that displayed significant growth, Jagged1 signaling through Notch2 suppressed proliferation; cultures with low growth potential were non-responsive to Jagged1. In addition, Jagged1 did not promote contractile smooth muscle actin nor have a significant effect on the mature differentiated phenotype. Thus, SMC derived from atherosclerotic lesions show distinct proteomic profiles and have altered Notch signaling in response to Jagged1 as a differentiation stimulus, compared with normal SMC.
\end{abstract}

Cardiovascular disease is the leading cause of death in western society and caused 17.3 million deaths worldwide

Electronic supplementary material The online version of this article (https://doi.org/10.1038/s41374-018-0072-1) contains supplementary material, which is available to authorized users.

$\triangle$ Lucy Liaw

liawl@mmc.org

1 Center for Molecular Medicine, Maine Medical Center Research Institute, 81 Research Drive, Scarborough, ME 04074, USA

2 Department of Developmental, Molecular and Chemical Biology, Sackler School of Graduate Biomedical Sciences, Tufts University School of Medicine, 136 Harrison Ave., Boston, MA 02111, USA in 2013, the great majority of which were ischemic in nature due to narrowing of the artery from atherosclerotic plaque. In the United States, the probability at birth that an individual will eventually die of cardiovascular disease is $47 \%$ [1]. Despite the high incidence of atherosclerosis, the complex cellular processes of plaque formation are still incompletely understood. What is generally accepted is that damaged endothelial cells allow lipid moieties and inflammatory cells to accumulate within the arterial wall resulting in progressive recruitment of other vascular and inflammatory cells over many years [2, 3].

Vascular smooth muscle cells (SMC) affect plaque formation in several ways; therefore, the signaling pathways that govern their behavior during atherosclerosis are of great interest. Hyperproliferation of vascular SMC create 
thickened medial walls, SMC form fibrous plaque caps by secreting extracellular matrix proteins and collagen, and even contribute to the necrotic core as macrophage-like foam cells $[4,5]$. The signaling that causes vascular SMC to switch from a quiescent, contractile phenotype to a highly proliferative, extracellular matrix depositing, migratory phenotype may be a valuable therapeutic target.

A few studies show that Notch regulates neointima formation after vascular injury [6-8], a process resulting from SMC proliferation and migration. Both Notch2 and Notch3 are expressed in vascular SMC, and it has been shown that Notch2 mediates quiescence in healthy human SMC [9]. Notch signaling is activated by a transmembrane ligand such as Jagged1, which induces proteolytic cleavage and translocation of the Notch intracellular domain (NICD) to the nucleus. NICD binds to the transcriptional repressor Recombining Binding Protein suppressor of hairless (RBP-J), causing displacement of co-repressors and recruitment of co-activators to initiate transcription of Notch/RBP-J downstream targets.

In atherosclerosis, Notch signaling has been implicated in inflammation, with Delta-like 4 (DLL4) ligand stimulation leading to proinflammatory gene profiles in macrophages $[10,11]$, and inhibition of DLL4 in vivo suppressing macrophage influx and atheroma progression in a mouse model $[12,13]$. In addition to immune cells, the endothelium is a target of Notch signaling during atherosclerosis. Notch1 is expressed in vascular endothelial cells (EC), although variably in humans [14]. In the mouse, a high-fat diet suppressed Notch1 transcript, and in vitro, human EC expression of Notch1 was suppressed by oxidized phospholipids [14]. There is little information about whether Notch signaling in SMC also contributes to atheroma formation. Indeed, even Notch receptor levels in SMC in atherosclerotic plaques has not been well studied during human disease progression. A better understanding of the cell-type-specific signaling of Notch in all cells of the vessel wall is required to consider potential athero-protective effects of Notch blockade [15, 16]. In the present study, we evaluated human atherosclerotic lesions for SMC, EC and inflammatory cell patterns of Notch localization, and derived several SMC populations from endarterectomy samples to study Notch signaling. Our findings describe a consistent pattern of Notch protein in SMC in advanced atherosclerotic lesions, and also suggest that Notch signaling in sub-populations of these SMC promotes a growth-inhibitory effect.

\section{Materials and methods}

\section{Collection of human tissue samples}

This study was evaluated and approved by the Institutional Review Board at Maine Medical Center. Patients scheduled for an endarterectomy procedure (JE-J and colleagues) were consented for the anonymized use of their endarterectomy specimens in this research. We collected both carotid artery and femoral artery endarterectomy specimens. In addition, we utilized the National Disease Research Interchange (http://ndriresource.org/) to obtain blood vessels from donors who did not have diagnosed cardiovascular disease. Exclusions for both endarterectomy patients and normal artery donors included any history of chemotherapy/radiation or sepsis.

\section{Vascular tissue processing}

Endarterectomy samples were collected in sterile phosphate-buffered saline (PBS) and stored at $4{ }^{\circ} \mathrm{C}$ prior to processing. The time from removal from patient to arrival at the lab was $\sim 1-3 \mathrm{~h}$. Samples were divided into several pieces. One piece from the area with largest visible plaque, as well as one region with no apparent plaque were collected and placed in 10\% formalin overnight for further processing and paraffin embedding. A second piece containing the plaque and adjacent tissue was frozen and stored at $-80{ }^{\circ} \mathrm{C}$ for future collection of protein and/or total RNA. In patients under the age of 70, a final piece of the endarterectomy sample was placed in culture for propagation by explant culture.

\section{Derivation of SMC populations}

Several procedures were tested for efficiency of primary explant into stable cell populations. In some cases, endarterectomy or normal vessel tissue was washed in PBS, minced and digested for $5 \mathrm{~min}$ at $37^{\circ} \mathrm{C}$ in $0.25 \%$ trypsin/ EDTA. The tissue pieces were then plated onto tissue culture dishes coated with $0.1 \%$ gelatin in SmGM2 human smooth muscle growth media (Lonza, medium contains 5\% serum, epidermal growth factor, fibroblast growth factor-2, insulin and antibiotics). Alternatively, tissue pieces were washed in PBS, cut into segments and directly plated in SmGM2 media on $0.1 \%$ gelatin-coated plates. In both cases, explanted cells were passaged onto new plates when cells became dense and the tissue was kept on the original plate to allow additional cells to migrate out as a separate population. Only first explant populations were used here. Both methods were successful in yielding primary populations of SMC. Depending on the specimen, cells were visible on the plate between 2 and 3 weeks after explant. Initial studies determined that no cultures were successfully derived from patients with advanced age, either due to lack of cell outgrowth or early senescence of explanted cells. Thus, subsequent studies focused on endarterectomy tissue derived from patients under the age of 70 . Cell populations that were successfully established in primary culture were 
cryopreserved at low passage, and cell pellets were frozen for protein lysates (immunoblot and mass spectrometry) or used for Notch signaling assays and immunofluorescence. For comparison, we also utilized commercially available normal human SMC derived from donor carotid artery (hCtASMC lot 2435, derived from a 33-year-old male donor, Cell Applications).

\section{Immunostaining of tissue sections}

Samples fixed overnight in $10 \%$ formalin were washed with PBS, and washed in 70\% ethanol. Calcified plaque samples were treated with $10 \%$ ethylenediaminetetraacetic acid (EDTA) at $\mathrm{pH}=7.0-7.4$ with the solution changed every 2-3 days until decalcification occurred. Samples were dehydrated in an automated tissue processor, transferred to AmeriClear $^{\mathrm{TM}}$ (CardinalHealth, Dublin, Ohio) and then to paraffin. Samples were embedded in paraffin for sectioning. Formalin-fixed, paraffin-embedded tissues were serially sectioned at $5 \mu \mathrm{m}$ and sections were rehydrated in Amer$\mathrm{iClear}^{\mathrm{TM}}$ followed by ethanol in preparation for hematoxylin/eosin, Masson's trichrome with Verhoeff's elastic stain, or immunohistochemical (IHC) staining. Slides for IHC underwent additional processing prior to staining: $0.01 \mathrm{M}$ sodium citrate antigen retrieval, endogenous peroxidase quenching with $3 \%$ hydrogen peroxide, permeabilization with $0.5 \%$ Triton $\mathrm{X}-100$, and blocking in $2 \%$ bovine serum albumin (BSA) and $2 \%$ goat serum. IHC sections were then incubated overnight with primary antibodies (see below) diluted in blocking solution. Primary antibodies were detected using SignalStain ${ }^{\circledR}$ Boost IHC Detection Reagent and SignalStain ${ }^{\circledast}$ diaminobenzidine substrate kit (Cell Signaling Technologies-CST, Danvers, MA) with hematoxylin counterstain. Sections were dehydrated with ethanol then AmeriClear ${ }^{\mathrm{TM}}$ before coverslipping. IHC primary antibodies include Notch1 (CST 3608, dilution 1:400), Notch2 (CST 4530, dilution 1:400), Notch3 (Abcam ab23426, dilution 1:500), SM-MHC (Millipore MAB3570, dilution 1:250) and CD45 (Abcam ab33533, dilution $1: 200)$.

\section{Jagged1/Notch assays}

Notch activation was achieved by plating cells on dishes pre-coated with $3 \mu \mathrm{g}$ recombinant rat Jagged1 fused to human $\mathrm{Fc}$ or with a human $\mathrm{Fc}$ control protein (R\&D Systems, Minneapolis, MN). Immobilization of control Fc or Jagged1-Fc was performed by incubating 6-well and 24well culture plates with $1 \mathrm{~mL}$ or $0.25 \mathrm{~mL}$ per well of 6.94 $\mathrm{pmol} / \mathrm{mL}$ Fc-specific human IgG (Sigma) dissolved in PBS for $2 \mathrm{~h}$ at room temperature. The solution was then aspirated and 6-well and 24-well plates were incubated with $1 \mathrm{~mL}$ or $0.25 \mathrm{~mL}$ per well of $16.68 \mathrm{pmol} / \mathrm{mL}$ purified $\mathrm{Fc}$ or recombinant rat Jagged1-Fc chimera dissolved in PBS for $72 \mathrm{~h}$ at $4{ }^{\circ} \mathrm{C}$. The solution was then aspirated before plating cells for $48 \mathrm{~h}$ of activation.

\section{Small interfering RNA knockdown}

In some experiments, small interfering RNAs or scrambled control (Qiagen, Hilden, Germany) were used to suppress Notch2 or Notch3 protein. Stocks of targeting siRNA or non-targeting control were diluted in serum-free medium to a concentration of $0.2 \mathrm{pmol} / \mu \mathrm{l}$. In a separate tube, a 1:20 dilution of Lipofectamine RNAiMAX Transfection Reagent (ThermoFisher Scientific, Waltham, MA) was prepared in serum-free medium. Equal volumes of the siRNA solution and the RNAiMAX solution were mixed together at room temperature for $10 \mathrm{~min}$. Cells were washed twice with PBS, and fed with antibiotic-free SmGM2 growth medium. The siRNA transfection reagent was added to the cells at a final concentration of $0.023 \mathrm{pmol} / \mu \mathrm{l}$ of siRNA. Cells were transfected for $24 \mathrm{~h}$, and then plated in the Jagged1/Notch assay in antibiotic-free SmGM2.

\section{Cell proliferation assays}

Proliferation of explanted cells was analyzed using the Click-iT ${ }^{\circledast}$ EdU imaging kit (Invitrogen C10337, Waltham, MA). Cells were plated on Jagged1-Fc and Fc controlcoated 24-well plates, allowed to incubate for $24 \mathrm{~h}$, and then treated with $10 \mu \mathrm{M}$ 5-ethynyl-2'-deoxyuridine (EdU) diluted in SmGM2 for an additional $24 \mathrm{~h}$. Cells were then fixed using $3.7 \%$ formaldehyde and permeabilized in $0.5 \%$ Triton $^{\circledR} \mathrm{X}-100$. Click-iT ${ }^{\circledast}$ EdU detection was performed, as well as nuclear staining with 4',6-diamidino-2-phenylindole (DAPI). At least three wells per treatment were each imaged in seven zones on a Leica DMIRB epi-fluorescent microscope (Wetzlar, Germany). Thresholding, watersheding and particle analysis was completed in ImageJ (NIH Bethesda, MD) for the DAPI channel and the EdU channel for each image so the ratio of EdU-positive cells to total cells could be calculated as a percentage.

\section{Proteomic analysis of endarterectomy-derived SMC populations}

hCtASMC and cells derived from carotid and femoral endarterectomy samples (Car23, Car31, Car45, Fem48 and Car56) were pelleted (starting material $\sim 1 \times 10^{6}$ cells) and stored at $-80^{\circ} \mathrm{C}$ prior to processing. Cells were lysed and proteins extracted using the Qproteome kit (Qiagen, Hilden, Germany). On ice, $10 \mu \mathrm{L}$ protease inhibitor plus $0.04 \mu \mathrm{L}$ benzonase nuclease was added to $1 \mathrm{~mL}$ of lysis buffer. Lysis solution $(180 \mu \mathrm{L})$ was then added to each tube, along with two magnetic beads. Samples were then homogenized at 
maximum speed for $60 \mathrm{~s}$ using an Autodisruptor. Tubes were centrifuged at $14,000 \mathrm{rpm}$ for $10 \mathrm{~min}$ at $4{ }^{\circ} \mathrm{C}$. The supernatant was transferred into a new tube with four volumes $(720 \mu \mathrm{L})$ of ice cold acetone. Tryptic digests of protein samples were performed using the ProteoExtract digestion kit (Calbiochem, Darmstadt, Germany). Tryptic peptides were then separated on an Ultimate RSLC system 3000 (ThermoFisher/Dionex, Waltham, MA) nanoscale liquid chromatograph and infused onto a 5600 TripleTOF mass spectrometer (Sciex, Framingham, MA). Sequential window acquisition of all theoretical spectra (SWATH) was used to profile all proteins in each sample using a dataindependent acquisition method [17]. A human-specific ion library comprising 4091 proteins was constructed using ProteinPilot software (Sciex, Framingham, MA). For identification of peptides, multiple fragment ion chromatograms were retrieved from the spectral library for each peptide of interest. These spectra were compared with the extracted fragment ion traces for the corresponding isolation window to identify the transitions that best identify the target peptide. SWATH analysis was performed using PeakView software, and MarkerView software was utilized for principal component analysis and $T$-test comparisons.

\section{Immunoblot analysis}

Cell lysates were collected by scraping in cold RIPA buffer with protease inhibitors. Tissue lysates were collected by vortexing tissue ground in liquid nitrogen in cold RIPA buffer with protease inhibitors followed by sonication. Following quantification of protein concentration, samples were run on 10 or $12 \% \mathrm{TGX}^{\mathrm{TM}}$ FastCast ${ }^{\mathrm{TM}}$ Acrylamide gels (Bio-Rad, Hercules, CA). Proteins were transferred to polyvinylidene difluoride membranes, blocked using a solution of $5 \%$ skim milk in PBS with $0.1 \%$ Tween-20 (PBS-T) and probed $48 \mathrm{~h}$ with primary antibodies diluted in the same solution. The following antibodies were used: Notch2 (CST 4530, dilution 1:2000), Notch3 (CTS 5276S, dilution 1:2000), smooth muscle-myosin heavy chain (SMMHC; Abcam Ab53219, dilution 1:4000), smooth muscle actin (SM actin; Sigma A2547, dilution 1:8000), calponin (Abcam Ab46794, dilution 1:2000), cyclophilin (Santa Cruz SC130626, dilution 1:2000) and $\alpha$-tubulin (CST 21445, dilution 1:2000). Membranes were incubated for $1 \mathrm{~h}$ at room temperature with 1:5000 horseradish peroxidase (HRP)-linked mouse or rabbit secondary antibodies (CST, Danvers, MA) diluted in 5\% skim milk in PBS-T followed by detection with Luminata ${ }^{\mathrm{TM}}$ Classico or Forte Chemiluminescent HRP substrate (MilliporeSigma, Billerica, MA). Imaging was performed on a ChemiDoc ${ }^{\mathrm{TM}}$ MP imaging system. Quantification was done using ImageJ (NIH Bethesda, MD) with integrated pixel density of bands normalized to cyclophilin or $\alpha$-tubulin.

\section{Immunofluorescence staining}

SM actin expression in explanted cells was analyzed by immunofluorescence. SM actin primary antibody (Sigma A2547, dilution 1:500) was diluted in 2\% BSA (EMD Chemicals, San Diego, CA) in PBS. Primary antibodies were detected using 1:500 Alexa Fluor ${ }^{\circledR}$ 568-conjugated mouse secondary antibody (Life Technologies A11004, Waltham, MA) and nuclei were detected with 1:10,000 DAPI (Life Technologies D1306) diluted in 2\% BSA in PBS. Imaging was performed on a Leica SP8 confocal microscope (Wetzlar, Germany) or a Leica DMIRB epifluorescent microscope (Wetzlar, Germany).

\section{Statistical analysis}

Immunoblot densitometry data from three technical replicate blots for non-Jagged stimulated endarterectomy and normal vessel-derived cells were used to calculate the relative levels of Notch2, Notch3, SM actin, SM-MHC and calponin protein in endarterectomy cell populations (Fig. 4b, c, Fig. 5b). When multiple normal cells populations were analyzed, the replicates for each normal cell population were averaged and included in the model as one observation per cell population under "NV grouped" in order to allow for comparison between the normal cells and each endarterectomy-derived cell population. The statistical significance of relative protein levels was calculated using one-way analysis of variance (ANOVA) followed by Dunnett's multiple comparisons test of each endarterectomy-derived cell population to the grouped normal vessel-derived SMC. Immunoblot densitometry data from three technical replicate blots for ground flash-frozen endarterectomy samples and normal vessels were used to calculate the relative levels of Notch2, Notch3, SM actin, SM-MHC and calponin protein (Fig. 5d) and statistical significance was calculated as above. Immunoblot densitometry data from two to five biological replicate blots for Jagged1-Fc-treated endarterectomy-derived and normal vessel-derived cells were normalized to Fc control data and used to calculate the relative levels of SM actin and SMMHC protein in the endarterectomy cell populations (Fig. 6a, b). As in the previous analysis, Fc control normalized replicates for each normal cell population were averaged and included in the model as one observation per cell population under "NV grouped". The statistical significance of Jagged1-Fc-mediated relative protein level was calculated using one-way ANOVA followed by Dunnett's multiple comparisons test of each endarterectomy-derived cell population to the grouped normal vessels. The statistical significance of Jagged1-Fc treatment on proliferation for each endarterectomy and normal vessel-derived cell population (Fig. 7a) was calculated using two-way ANOVA 
with cell population and Jagged1-Fc treatment as factors followed by Sidak's multiple comparisons test of treated versus non-treated within each cell population. In immunoblots to validate siRNA targeting (Fig. 7b), cyclophilin normalized Notch2 and Notch3 levels from siN2 and siN3treated cells were normalized to NT-treated cells. Four twoway ANOVAs with cell population and siRNA treatment as factors were performed comparing siN2 and siN3 treated to NT treated within each cell population. Dunnett's multiple comparisons test was used for post hoc analysis. The degree of Jagged1-Fc-mediated suppression of proliferation was calculated for endarterectomy-derived and normal vesselderived cell populations after treatment with non-targeting, Notch2 targeting or Notch3 targeting siRNA (Fig. 7c). Statistical significance of suppression was calculated using two-way ANOVA with cell population and siRNA treatments as factors followed by Dunnett's multiple comparisons test of Notch2 and Notch3 siRNA to non-targeting siRNA for each cell population. This effect was further analyzed for differences between endarterectomy and normal vessel-derived primary cultures by a mixed ANOVA model with a fixed effect of cell population type (endarterectomy or normal vessel-derived), a random effect of cell population nested within cell population type, and a fixed effect of siRNA treatment. Results are shown as normalized means \pm the standard error of the mean (SEM). Differences were considered statistically significant at $p<0.05$. All analyses were performed in GraphPad Prism 7.04 except for the mixed ANOVA model, which was performed in SAS version 9.2.

\section{Results}

Our study included specimens from patients undergoing carotid or femoral endarterectomy at Maine Medical Center (Table 1). Subjects had a median age of 68 years. Endarterectomy was performed less frequently in females than in males, but when it was performed, it was done so at an earlier age with a median age of 62 years for females compared with a median age of 70 years for males. Carotid endarterectomy is much more common than femoral endarterectomy at our site, especially in females. We noted that plaque samples derived from femoral endarterectomy procedures were more calcified than the majority of samples derived from carotid endarterectomy procedures. Due to the relatively low number of femoral endarterectomy patients in our study, however, we were unable to quantify characteristics that were unique to femoral arteries versus carotid artery tissues.

To compare features of SMC from atherosclerotic lesions to healthier vessels, we obtained carotid arteries from donors without diagnosed cardiovascular disease (Fig. 1).
Table 1 Demographics for endarterectomy patients

\begin{tabular}{ll}
\hline Gender & \\
Male & $58 \%$ \\
Female & $42 \%$ \\
Median age & \\
Total & 68 years \\
Male & 70 years \\
Female & 62 years \\
Plaque location (carotid/femoral) & \\
Total & $82,18 \%$ \\
Male & $78,22 \%$ \\
Female & $91,9 \%$ \\
\hline
\end{tabular}

Although some of these arteries had significant levels of plaque formation (data not shown), we found several with relatively low levels of intima, as defined by tissue on the luminal side of the internal elastic lamina. Comparisons of structure between full donor arteries and surgically resected plaques show highly enlarged intima/plaque in the endarterectomy patients.

Both Notch2 and Notch3 are known regulators of SMC phenotype, although they have not been well characterized in human atherosclerotic lesions. Vascular tissue from atherosclerotic plaques derived from human endarterectomy was immunostained to detect Notch2 and Notch3 protein. We focused on discrete regions of the vessel, including medial SMC (Fig. 2a), intima/plaque (Fig. 2b), regions of fibrous cap (Fig. 2c) and medial/intimal transition regions (Fig. 2d). We surveyed $>50$ endarterectomy samples, and utilized additional markers to detect populations of SMC (SM-MHC) and inflammatory cells (CD45) in adjacent sections. Qualitative data scoring of protein levels in these vascular compartments and cell types are shown for all samples in Supplemental Table 1. We found a consistent pattern of protein localization in human atherosclerotic lesions (Supplemental Table 2). Notch2 was observed in SMC of the media and intima, although at lower levels in the intima/plaque (Fig. 2a, b). Notch3 protein was abundant in medial and intimal SMC (Fig. 6a, b), and both Notch2 and Notch3 protein localized to regions of fibrous cap (Fig. 2c). Notch3 protein localization had a distribution that was similar to SM-MHC, but more extensive in intimal SMC regions (Fig. 2d). As SMC contractile proteins, such as SM-MHC, are known to be modulated in diseased SMC, our observations suggested that Notch3 may be a more stable marker of SMC within human atherosclerotic plaques.

By contrast, Notch1 protein was not found in medial or intimal SMC within human atherosclerotic lesions. However, Notch1 was sporadically expressed in inflammatory cells (Fig. 3b, c), and was a strong marker of EC in vasa 


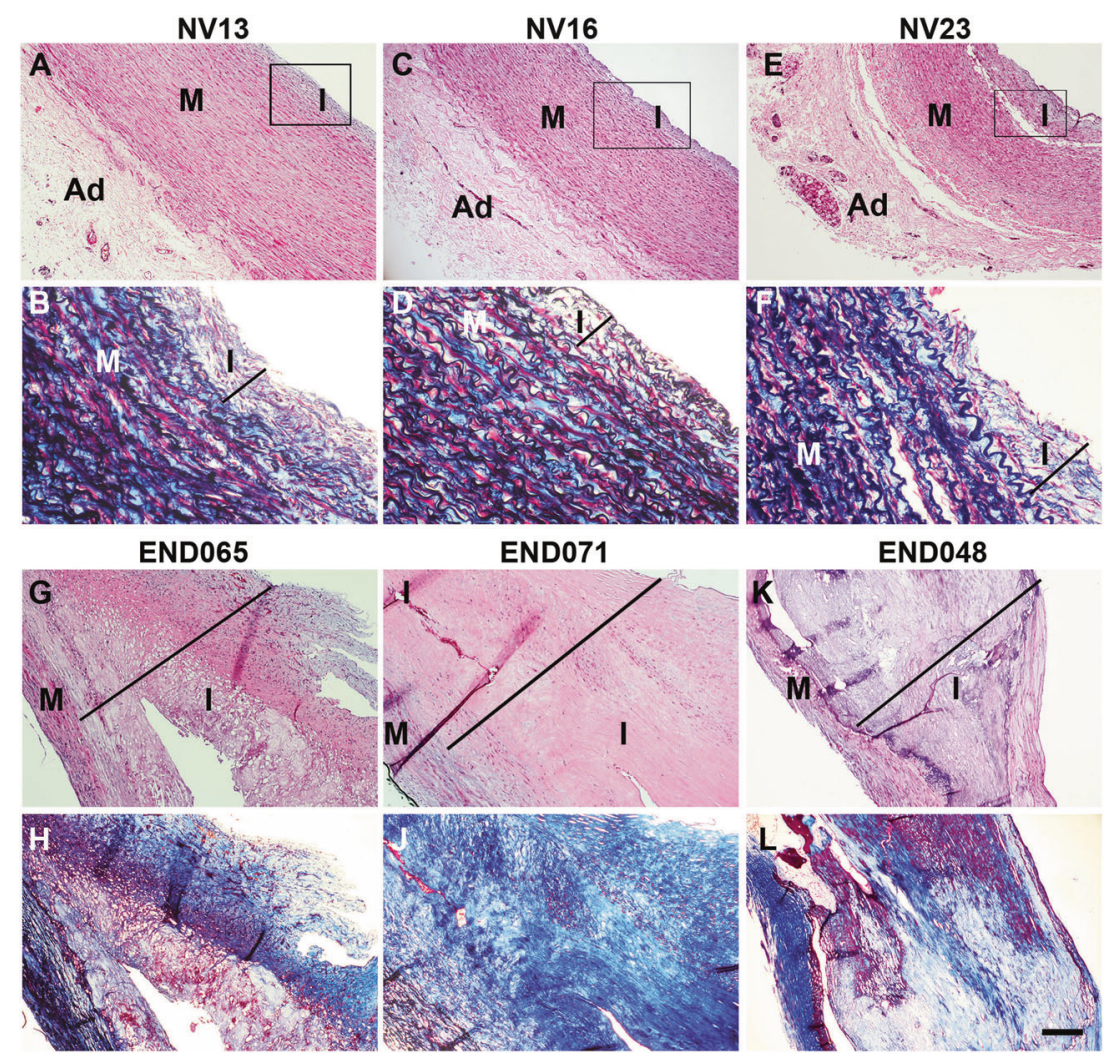

Fig. 1 Characteristics of human vascular tissue from donors without cardiovascular disease or with advanced atherosclerosis. Examples show samples from three carotid artery donors with no diagnosed cardiovascular disease (donors of normal vessels 13, 16 and 23), and samples from three donors with advanced cardiovascular disease requiring surgical intervention (carotid endarterectomy patients 065 and 071 , and femoral endarterectomy patient 048). Tissue sections were stained with hematoxylin/eosin (a, $\mathbf{c}, \mathbf{e}, \mathbf{g}, \mathbf{i}, \mathbf{k})$ or trichrome/ Verhoeff's stain. b, d, f Show trichome/Verhoeff's staining at a higher

vasorum and lumenal ECs (Fig. 3d, e). Notch2 was also found in inflammatory cells and EC, whereas Notch3 was only found in SMC underlying the endothelium (Fig. 3).

For Notch signaling studies, SMC populations were derived by explant culture from the endarterectomy samples. We also derived populations from healthy vessels NV13, NV16 and NV23 and obtained normal hCtASMC from a commercial source. By passage two or three, each cell population appeared homogenous, and several cell populations were successfully expanded for further signaling studies (Fig. 4a). Table 2 shows donor characteristics of explanted populations. Proliferative rates and maximum passage number varied between cell populations, likely based on patient or donor age and health. To verify that explanted populations were SMC, we analyzed levels of Notch2, Notch3 and smooth muscle markers through immunoblot (Fig. 4b, c). These markers were detected in most SMC populations, with variability in steady-state protein levels. The immunoblot data are consistent with the vascular in vivo staining within the SMC. Of note, the magnification of the boxed regions in $\mathbf{a}, \mathbf{c}, \mathbf{e} . \mathbf{h}, \mathbf{j}, \mathbf{l}$ Show trichome/ Verhoeff's staining of sections adjacent to those pictured in $\mathbf{g}, \mathbf{i}$, j. Intima (I) widths are marked by a solid black line with the boundary between intima and media (M) defined at the internal elastic lamina. Vessels from healthy donors show adventitia (Ad), whereas endarterectomy specimens do not as that portion of the vessel is not removed from the patient. Scale bar is $50 ? \mu \mathrm{m}$ in $\mathbf{b}, \mathbf{d}$ and $\mathbf{f}$, and 200 ? $\mu \mathrm{m}$ in the other panels

endarterectomy procedure removes only a portion of the medial wall, thus no adventitial fibroblasts are included in our samples.

To determine molecular features of the derived populations, we performed SWATH analysis after mass spectrometric identification of proteins from cell lysates. Several endarterectomy-derived populations were compared with normal human carotid artery SMC primary culture. Principal component analysis indicated that cell populations from carotid or femoral endarterectomy samples (Car23, Car31, Car45, Fem48 and Car56; Fig. 5a) differ from the normal carotid artery SMC. In order to determine how closely the explanted cell populations reflect their original tissue environment, we analyzed Notch2, Notch3 and smooth muscle markers through immunoblot of ground flash-frozen normal vessels (NV13, NV16 and NV23) and endarterectomy samples (End31, End45, End48 and End56), as well as normal carotid artery SMC and cells explanted from endarterectomy samples (Car31, Car45 and Car56; Fig. 5b, c). When endarterectomy-derived samples were 


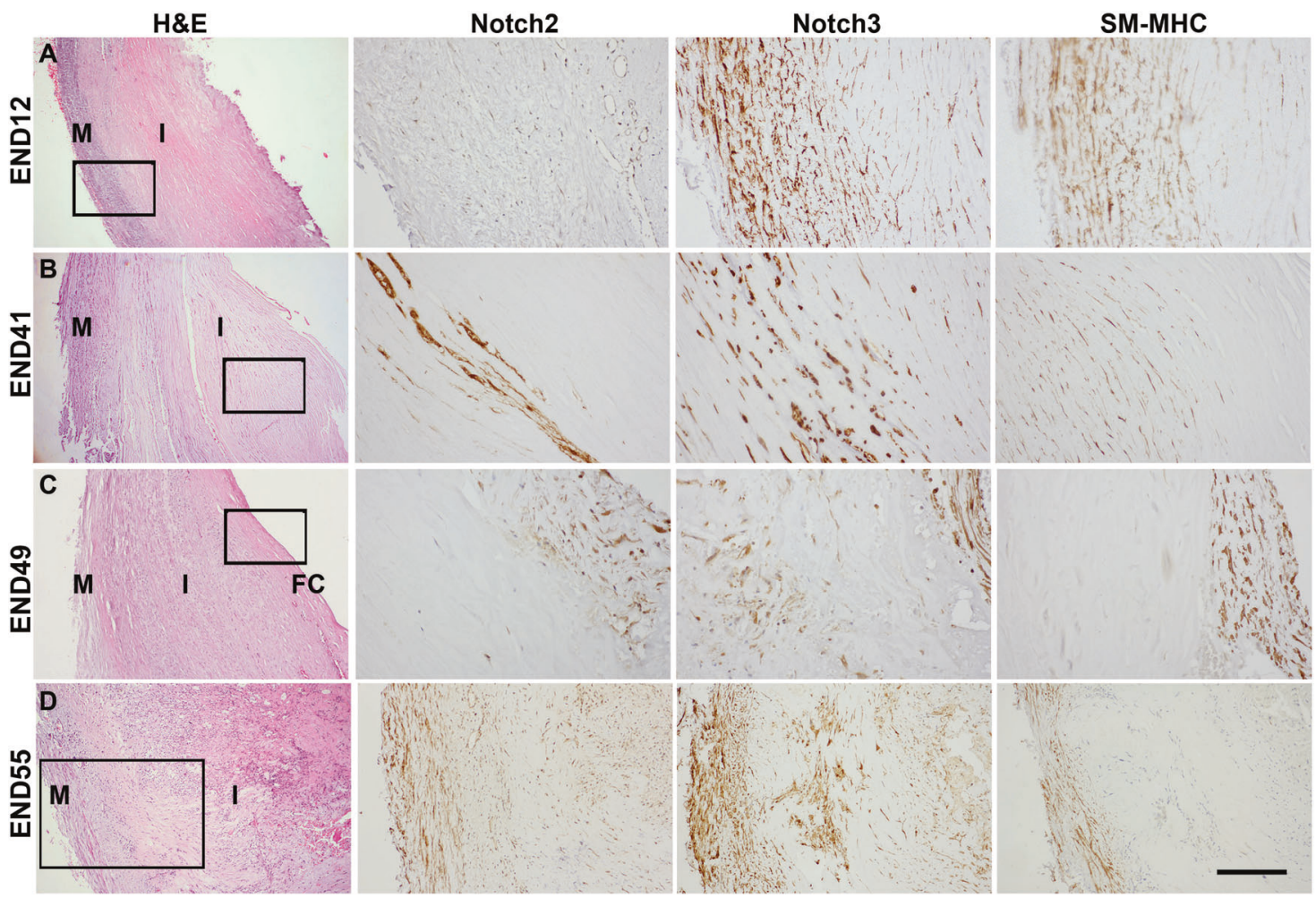

Fig. 2 Notch localization in diseased vessels. Serial tissue sections from endarterectomy patients 12 (a, carotid), 41 (b, carotid), 49 (c, femoral) and 55 (d, carotid) were immunostained to localize Notch2, Notch3 or SM-MHC proteins. Normal IgG was used as a negative control. The lumens of vessels are to the upper right, itima (I),

compared with normal vessel-derived samples, smooth muscle markers were at similar or higher levels in both tissue and explant samples. Similarly, both Notch2 and Notch3 were detected in all SMC primary cultures. In human lesions, Notch2 was undetectable in all tissue lysates, consistent with the faint immunostaining detected in the plaque sections. However, Notch3 protein was present in tissue lysates from healthy vessels and endarterectomy samples (Fig. 5d, e). Smooth muscle markers were also detected at similar levels in healthy vessels and plaques.

As a second method to test the phenotype of the endarterectomy-derived populations from donor lesions, we determined how similar the average proteome from five plaque-derived populations (Car31, Car45, Fem48, Car23 and Car56) was to the normal human arterial SMC population. We obtained $\sim 1000$ proteins for comparison, out of which 855 were not significantly different when comparing the plaque-derived populations in aggregate with the normal carotid artery SMC. Given that the molecular profile of SMC is rich with proteins associated with the cytoskeleton that mediate contractile function and signaling, we identified the shared proteins that are categorized as structural molecules in the PANTHER classification system [18]. Our list of shared proteins in this category (Supplemental media (M) and fibrous cap (FC) are marked. Images of immunostained tissue are magnified from the boxes in H\&E images on left side of figure. Scale bar for immunostained images is $100 ? \mu \mathrm{m}$ in panels a-c and $200 ? \mu \mathrm{m}$ in panel $\mathbf{d}$

Table 3) includes those that have previously been described as defining SMC [19], but also contains unique proteins. Despite these similarities, there were also a significant number of proteins (133) that were lower in the populations derived from endarterectomy tissue compared with normal SMC (Supplemental Table 4), but only 22 proteins in aggregate were higher in endarterectomy-derived populations compared with normal SMC (Supplemental Table 5).

The successful derivation of endarterectomy-derived SMC provided an opportunity to understand the effect of Notch signaling in diseased SMC. We previously showed that Notch signaling stimulated by Jagged 1 in healthy SMC induces differentiation and the expression of contractile markers via both Notch2 and Notch3, and suppresses proliferation via Notch2 [9]. However, Notch signaling activity has not been tested in SMC from patients with advanced cardiovascular disease. We first analyzed effects of Jagged1-Fc on SMC contractile markers in endarterectomyderived SMC populations (Fig. 6). As we previously described $[9,20,21]$, stimulation of healthy SMC with Jagged1-Fc increased levels of SM actin (Fig. 6). We also tested eight endarterectomy-derived SMC populations (seven from carotid arteries and one from a femoral artery). Unlike SMC from donors with no vascular disease 


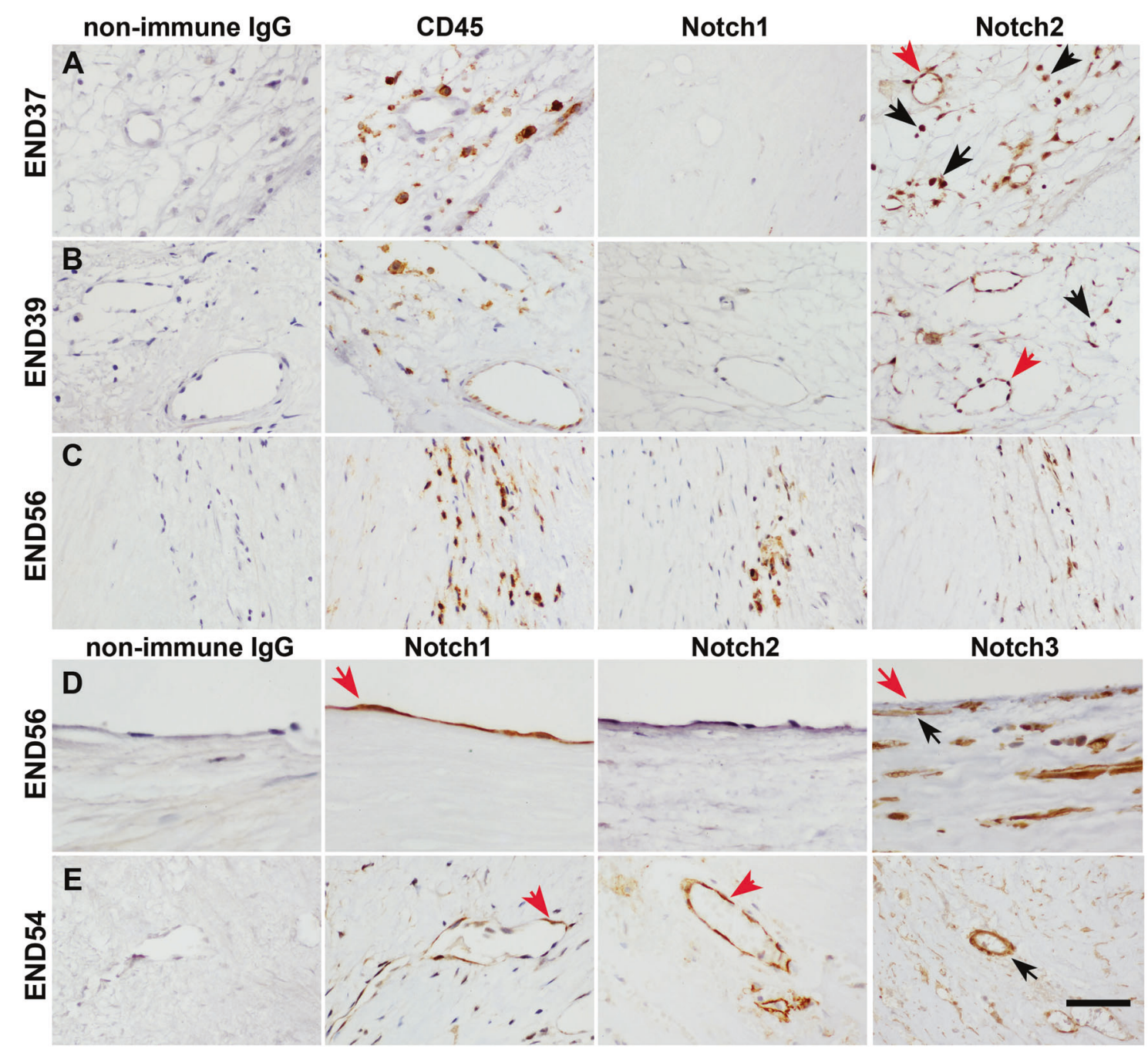

Fig. 3 Localization patterns of Notch in inflammatory and endothelial cells. Serial tissue sections from carotid endarterectomy patients $37 \mathbf{a}$, $39 \mathbf{b}, 56 \mathbf{c}, \mathbf{d}$ and 54 e were immunostained to localize CD45, Notch1, Notch 2 or Notch3. Notch 2 frequently colocalized with CD45-positive inflammatory cells (black arrowheads). Notch1 and Notch2 often colocalized with endothelial cells (red arrowheads). Notch3 was localized to SMC underlying endothelium (black arrowhead in rightmost image for panel $\mathbf{d}$ and SMC in vasa vasorum in rightmost image for panel e. Normal IgG was used as a negative control. Scale bar is $25 \mu \mathrm{m}$ in panel $\mathbf{d}$ and $50 \mu \mathrm{m}$ in all others
(hCtSMC, NV13 and NV23), Jagged1-Fc did not promote the contractile phenotype in SMC derived from endarterectomy samples, as measured by SM actin and SM-MHC levels. Immunofluorescence staining to detect SM actin confirmed our immunoblot results (Fig. 6c). Prior to Notch activation in the healthy SMC by Jagged1-Fc, SM actin expression was low or restricted to a small portion of the population. Upon Notch activation, SM actin expression was visible in a larger proportion of the population for healthy SMC, whereas remaining unchanged in SMC derived from diseased arteries.

We next compared proliferation of healthy SMC (hCtASMC, NV13 and NV23) populations versus endarterectomy-derived SMC populations (Fig. 7a). We previously observed that Jagged1 suppresses proliferation in
SMC derived from healthy donors via Notch2 [9]. Analysis of four populations of SMC from healthy donors (hCtASMC, NV13, NV16 and NV23) confirmed that Jagged1-Fc stimulation suppressed proliferation. However, we found that endarterectomy-derived SMC responses to Jagged1-Fc fell into two categories-those that suppressed proliferation after Jagged1-Fc stimulation (Car31, Car025, Car058, Car065 and Car071), and those that were nonresponsive (Car30, Fem48, Fem50, Car012, Fem048, Car050, Car057, Car060, Car066 and Car070). Interestingly, with the exception of Fem48, the populations that were unresponsive to the Jagged1 quiescence signal tended to have low levels of basal proliferation. These data suggest that Notch activation within diseased SMC during phases of hyperproliferation may be effective to promote a quiescence 

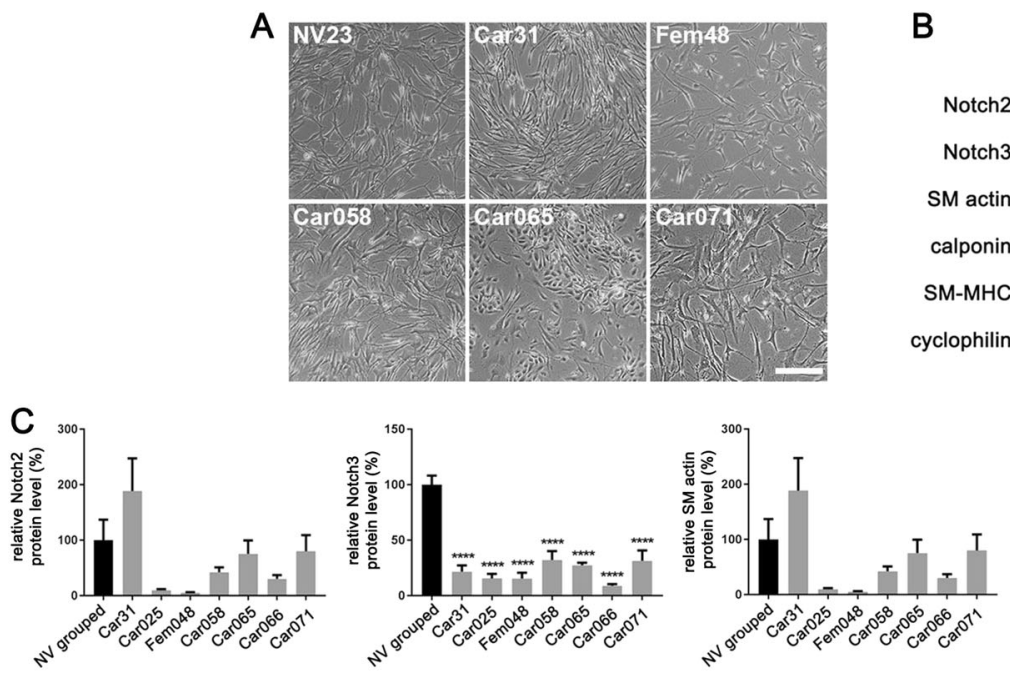
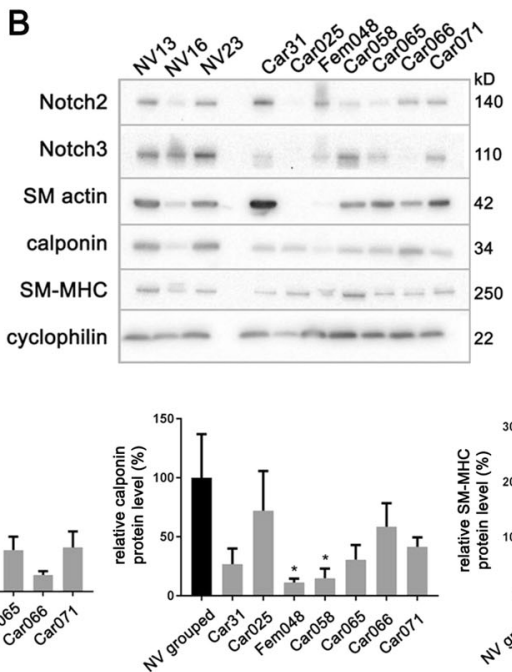

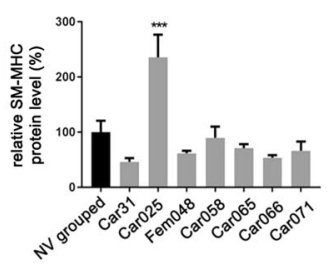

Fig. 4 Characteristics of SMC derived from endarterectomy specimens. a Phase micrographs showing the morphology of primary cultures successfully derived from a donor with normal vasculature (NV23) versus five endarterectomy-derived cultures. Scale bar $=250 \mu \mathrm{m}$. b Explanted cell populations were interrogated by immunoblot for the proteins indicated. c Whole-cell lysates were analyzed by sodium-dodecyl-sulfate polyacrylamide gel electrophoresis and immunoblotted for the indicated proteins with densitometry

Table 2 Patient and donor characteristics of explanted samples

\begin{tabular}{llll}
\hline Sample ID & Gender & Age (years) & Tissue location \\
\hline NV13 & Male & 50 & Carotid \\
NV16 & Female & 28 & Carotid \\
NV23 & Male & 1 month & Carotid \\
Car31 & Male & 59 & Carotid \\
Car45 & Female & 66 & Carotid \\
Fem48 & Male & 64 & Femoral \\
Fem50 & Female & 60 & Femoral \\
Car56 & Female & 60 & Carotid \\
Car012 & Male & 69 & Carotid \\
Car025 & Female & 57 & Carotid \\
Fem048 & Male & 68 & Femoral \\
Car050 & Male & 70 & Carotid \\
Car057 & Male & 56 & Carotid \\
Car058 & Female & 60 & Carotid \\
Car060 & Female & 54 & Carotid \\
Car065 & Male & 68 & Carotid \\
Car066 & Male & 56 & Carotid \\
Car070 & Female & 62 & Carotid \\
Car071 & Male & 63 & Carotid \\
\hline
\end{tabular}

signal, but that during phases of low growth, endarterectomy-derived SMC may be refractory to Jagged1 growth control.

In order to distinguish which SMC expressed Notch receptor is primarily responsible for mediating proliferation quantification. Cyclophilin normalized protein levels in endarterectomy-derived cell populations were normalized to the average level in the grouped normal vessels (NV grouped) and oneway ANOVA were performed comparing endarterectomy lines to NV grouped. Significant results of Dunnett's multiple comparisons test are indicated by asterisks: $* p \leq 0.05, * * * p \leq 0.001$, and $* * * * p \leq 0.0001$. Whiskers indicate SEM

in our cell populations, we knocked down Notch2 or Notch3 protein using siRNA. We confirmed suppression of protein by immunoblot analysis of cells treated with nontargeting (NT), Notch2 (siN2) or Notch3 (siN3) targeting siRNA, with or without Jagged1-Fc stimulation (Fig. 7b and supplemental Fig. 1a). In the two normal and three endarterectomy-derived populations tested, we found that compared with NT, siN2 treatment on average reduced Notch 2 levels by $89 \%$ without Jagged1-Fc stimulation and by $66 \%$ with stimulation. We did observe a downregulation of Notch3 protein with siN2 treatment, particularly with Jagged1-Fc stimulation. Because this was consistent using two siN2 sequences and because protein turnover is accelerated with Jagged1-Fc treatment, we predict that this may be due to a regulatory feedback loop. For siN3 treatment compared with NT, average reduction in Notch3 was $>90 \%$ with or without Jagged1-Fc stimulation. We did not observe downregulation of Notch2 protein with siN3 treatment. We then analyzed the endarterectomy-derived SMC populations to determine if Notch2 or Notch3 was mediating Jagged1induced suppression of proliferation. We compared Jagged1 signaling when Notch receptors were intact (NT treatment) to individual knockdown of Notch2 or Notch3 (Fig. 7c). We found that Notch2 was important for suppression of proliferation by Jagged1 in endarterectomyderived SMC, similar to SMC from donors without cardiovascular disease. To confirm specificity, we tested two Notch2 siRNA sequences, and two Notch3 siRNA sequences (Supplemental Fig. 1b). Overall, our results show 

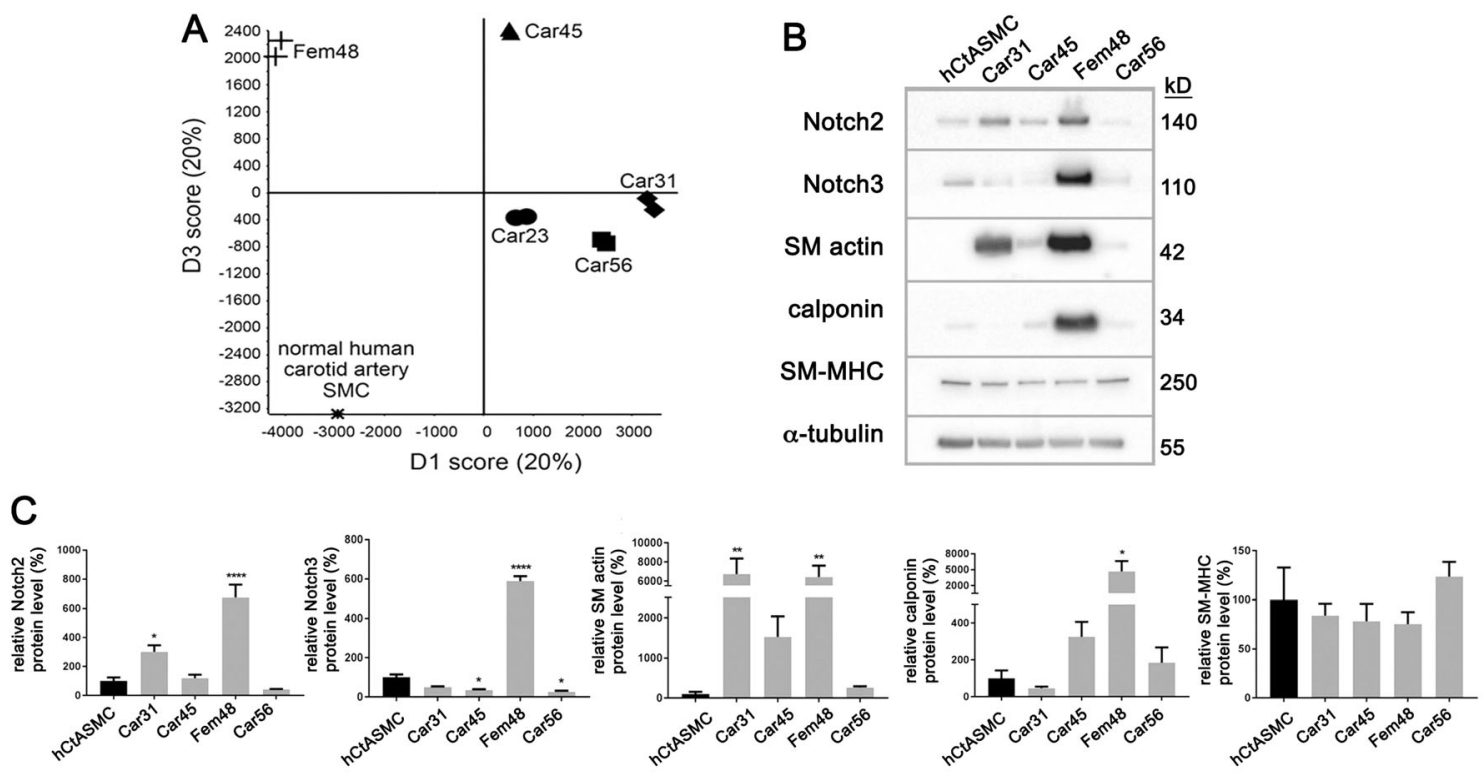

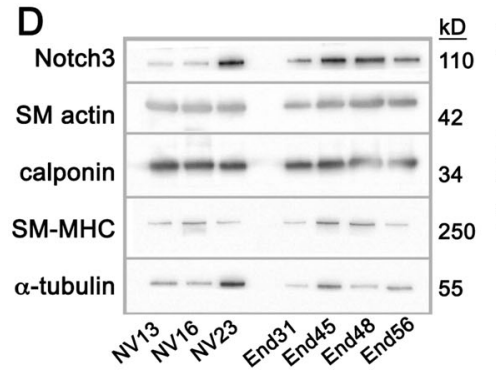

$E$
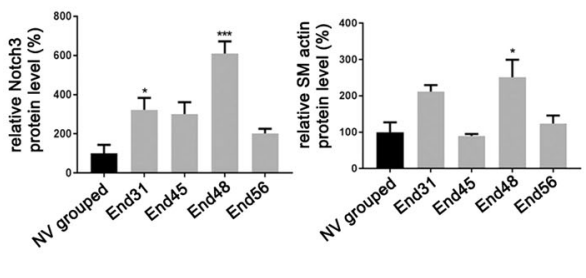

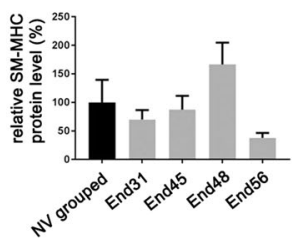

Fig. 5 Proteomic and targeted protein analysis in SMC derived from endarterectomy specimens. a SWATH analysis was performed on normal human carotid artery SMC versus the endarterectomy-derived populations and principal component analysis performed. b SWATH analyzed cell populations were further interrogated by immunoblot for the proteins indicated. c $\alpha$-Tubulin normalized protein levels in endarterectomy-derived cell populations were normalized to hCtASMC and one-way ANOVA were performed comparing endarterectomy lines with hCtASMC. Significant results of Dunnett's multiple comparisons test are indicated by asterisks: $* p \leq 0.05$,

that the Jagged1-Notch2 signaling axis is conserved in endarterectomy-derived SMC as a mechanism to promote cellular quiescence, and that particular sub-populations of diseased SMC are more responsive. In our analysis, we found a correlation of cells with higher growth potential with Jagged1 sensitivity. However, Jagged1 was unable to consistently increase the differentiated contractile phenotype of SMC populations from diseased vessels, suggesting alteration in this aspect of Notch signaling in SMC during atherogenesis.

\section{Discussion}

Notch signaling has been implicated in atherosclerotic disease progression, and has primary effects on EC, inflammatory cells and SMC. However, Notch signaling in SMC
$* * p \leq 0.01$ and $* * * * p \leq 0.0001$. Whiskers indicate SEM. d Tissues associated with SWATH analyzed cell populations were further interrogated by immunoblot for the proteins indicated. e $\alpha$-Tubulin normalized protein levels in endarterectomy samples were normalized to the average level in the grouped normal vessels (NV grouped) and one-way ANOVA were performed comparing endarterectomy lines to NV grouped. Significant results of Dunnett's multiple comparisons test are indicated by asterisks: $* p \leq 0.05$ and $* * * p \leq 0.001$. Whiskers indicate SEM

during atherosclerosis is not well understood. Given the accumulating evidence that Notch signaling impacts multiple cell types in the atherogenic vascular environment, our goal was to study human atherosclerotic plaques to (1) understand the landscape of Notch protein localization, particularly Notch 2 and Notch3, in human atherosclerotic SMC, (2) use endarterectomy-derived SMC to gain a better understanding of how SMC derived from diseased vessels are molecularly distinct from normal SMC and (3) understand how Notch signaling regulates SMC phenotype during disease.

Our initial study of 55 human endarterectomy samples defined a relatively consistent pattern of Notch protein within human plaques. Notch1 was almost exclusively localized to EC, although in some samples, expression in leukocytes was noted. Endothelial Notch1 was previously shown to be suppressed by a high-fat diet in the mouse, and 

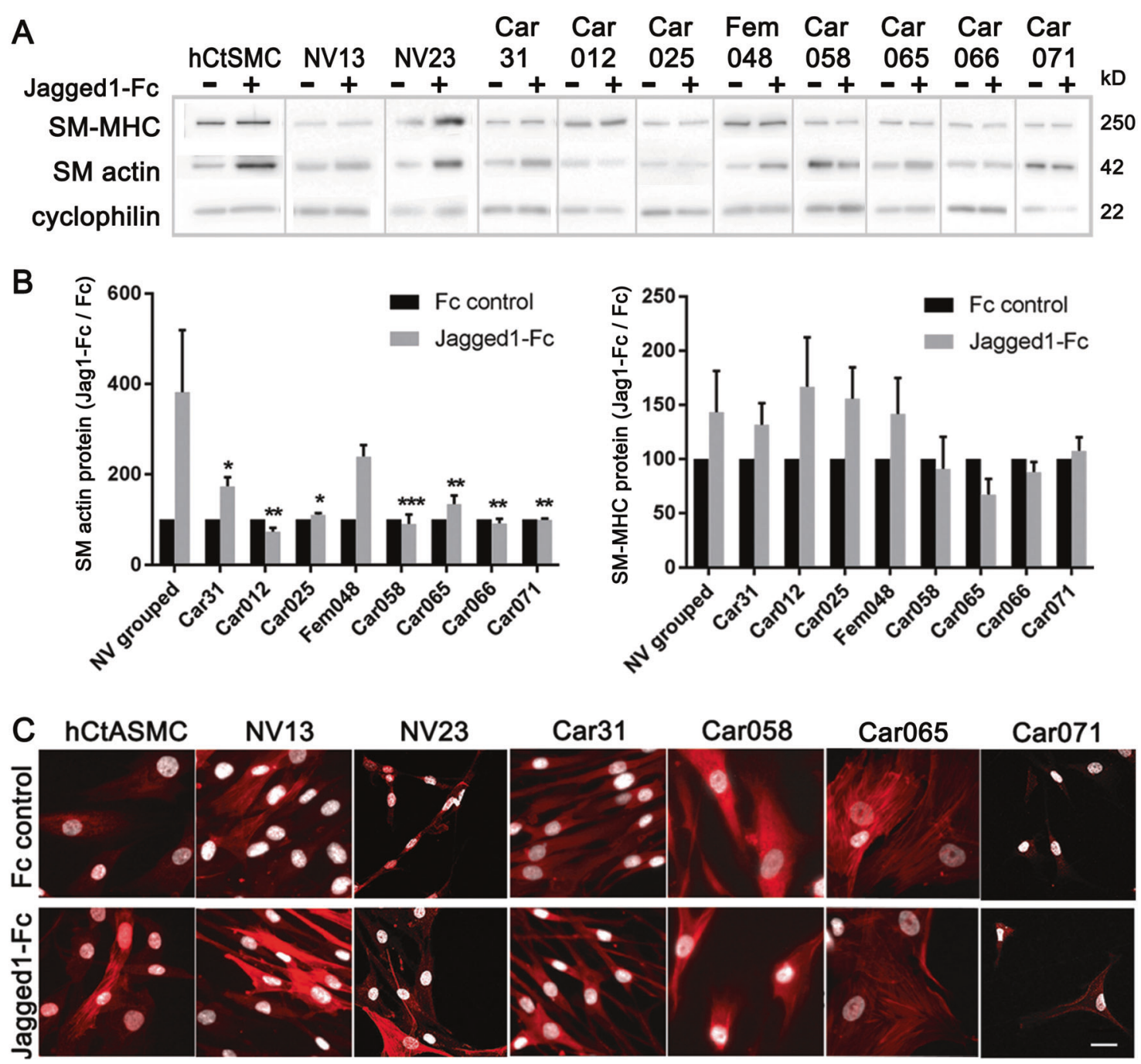

Fig. 6 Jagged 1 does not regulate contractile markers in endarterectomy-derived SMC. a immunoblot showing cell populations that were treated with Fc control (-) or Jagged1-Fc (+). b Cyclophilin normalized SM-MHC and SM actin levels from Jagged1-Fc stimulated groups in panel a were normalized to Fc control groups and one-way ANOVA were performed comparing endarterectomy populations with

suppressed in human aortic EC treated with inflammatory lipids (oxidized 1-palmitoyl-2-arachidonyl-sn- glycero-3phosphorylcholine) [14]. Our endarterectomy donors included patients with hyperlipidemia in whom levels of endothelial Notch1 may be regulated by lipid levels. In our endarterectomy samples, particularly strong levels of Notch1 were found in the endothelium of vasa vasorum. Jagged1/Notch1 signaling promotes leukocyte infiltration, thus promoting atherogenesis $[22,23]$. Notch 2 and Notch 3 protein were localized in EC and SMC of the media and the plaque. It has been shown that inflammatory cytokines trigger reduced Notch4 activity and increased Notch2 activity in EC promoting apoptosis and EC dysfunction [24]. Staining to detect leukocytes (CD45) showed that
NV grouped. Significant results of Dunnett's multiple comparisons test are indicated by asterisks: $* p \leq 0.05, * * p \leq 0.01$, and $* * * p \leq 0.001$. Whiskers indicate SEM. c Immunofluorescence staining of cells for SM actin in the presence or absence of Jagged1 stimulation. Scale bar $=50 \mu \mathrm{m}$

Notch 2 or Notch 3 is occasionally localized in inflammatory cells. Expression of Notch in inflammatory cells has been linked to reduced inflammatory response through suppression of Toll-like receptor signaling in macrophages [25] but has also been linked to proinflammatory responses in macrophages, as well as reduced diabetic wound healing $[26,27]$. Notch 3 consistently overlapped with areas of SMMHC staining, and also had a more widespread localization within SMC of the atherosclerotic lesion. Downregulation of Notch3 is associated with pathological vascular remodeling $[28,29]$ and thus high levels of Notch3 in certain areas of the plaque may reflect a stable lesion.

SMC explanted from endarterectomy samples can provide valuable insight into the changes in cellular processes 

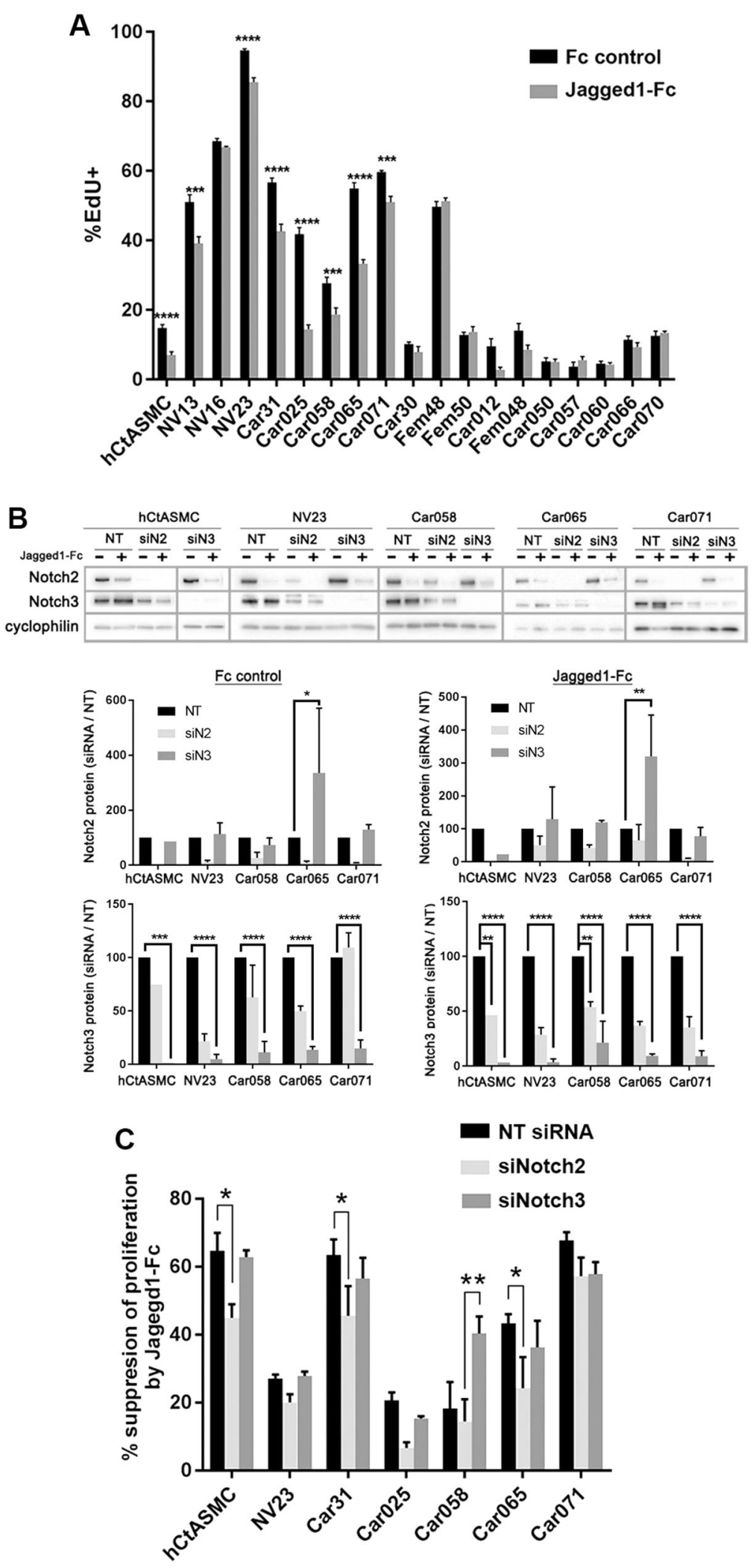
4 Fig. 7 Effects of Jagged1 on SMC proliferation. a Cells were treated with $\mathrm{Fc}$ control or Jagged1-Fc, and after $24 \mathrm{~h}$, labeled with EdU for an additional $24 \mathrm{~h}$. EdU-positive cells were quantified as a percentage of total cells for a given field and a two-way ANOVA was performed with cell population and Jagged1-Fc treatment as factors. Significant results of Sidak's multiple comparisons test of treated versus non-treated within each cell population are indicated by asterisks: $* * * p \leq 0.001$ and $* * * * p \leq 0.0001$. Whiskers indicate SEM. b Immunoblot demonstrating efficiency/specificity of Notch knockdown in cell populations that were transfected with a control non-targeting siRNA (NT), or siRNA to target Notch2 (siN2) or Notch3 (siN3) and densitometry quantification graphs. Cyclophilin normalized Notch2 and Notch3 levels from siN2 and siN3treated cells were normalized to NT-treated cells and two-way ANOVA with cell population and siRNA treatment as factors were performed comparing siN2 and siN3 treated with NT treated within each cell population. Significant results of Dunnett's multiple comparisons test are indicated by asterisks: $* p \leq 0.05, * * p \leq 0.01, * * * p \leq 0.001$ and $* * * * p \leq 0.0001$. Whiskers indicate SEM. c Cell populations with siRNA-mediated suppression of Notch 2 or Notch3 were used to identify the receptor required for the growth suppressive effect of Jagged1. Percent suppression of proliferation by Jagged1-Fc treatment was calculated and a two-way ANOVA with cell population and siRNA treatments as factors was performed comparing siN2 and siN3 treated with NT treated within each cell population. Significant results of Dunnett's multiple comparisons test are indicated by asterisks: * $p \leq 0.05$ and ${ }^{* *} p \leq 0.01$. Whiskers indicate SEM. A mixed ANOVA model with a fixed effect of cell population (endarterectomy or normal vesselderived), a random effect of cell population nested within cell population type, and a fixed effect of siRNA treatment was also performed and no difference was found between normal vessel and endarterectomyderived cells lines in their need for Notch2 to suppress proliferation

induced by atherosclerotic plaque. A few previous studies have addressed differences in SMC from normal or diseased donors. Pessi et al. [30] found that inducing inflammation in SMC from carotid endarterectomy specimens or normal human aortic SMC resulted in differential expression of a panel of miRNAs important in vascular cell phenotype, including inflammatory and adhesion molecules. Although these observations are intriguing, there are known differences in gene expression in endothelium derived from different anatomical sites [31, 32], or even adjacent EC within the same vessel [33]. As the majority of our endarterectomy samples were derived from carotid artery plaque, our analysis included normal SMC derived from presumably disease-free carotid arteries. Another study compared medial SMC from minimally diseased endarterectomy samples with medial SMC from the putative plaque region of the same samples [34]. This study found that diseased media were unable to produce SMC of the mature phenotype, whereas minimally diseased media displayed both mature SMC and migratory/proliferative SMC.

To further understand the molecular basis of diseased SMC, we performed SWATH proteomic analysis, which is an effective method of generating an unbiased view of cellular protein signatures. As more complete libraries of normal and diseased cell and tissue profiles are developed, we will gain a better understanding of which differentially expressed molecules are important in the disease state.
Proteomic analysis of extracellular matrix proteins by Langley et al. showed a promising avenue of distinguishing between patients with stable plaques and patients with vulnerable plaques [35]. This study analyzed tissue lysates from six carotid endarterectomy specimen from patients with acute ischemic stroke, and six asymptomatic individuals undergoing elective carotid endarterectomy. Identified biomarkers that were associated with the ischemic stroke patients include MMP9, CH13L1, S100A8, S100A9, cathepsin B, fibronectin and galectin-3 binding protein. Although our experiment was very different due to the derivation of primary SMC from each endarterectomy prior to proteomic analysis, we looked for similarities between these biomarkers and the results of our SWATH analysis. In our SMC, we detected cathepsin B, which was significantly decreased in all endarterectomy-derived SMC populations compared with normal SMC (Supplemental Table 4, average of 10-fold decrease in endarterectomy-derived SMC). Our study provides novel data using SWATH analysis to define common features of primary cultures of carotid artery SMC (including structural proteins, Supplemental Table 3), and identifies a subset of $\sim 15 \%$ proteins that distinguish normal SMC from endarterectomy-derived SMC. These analyses considered SMC populations derived from five patients, and thus the changes described are consistent and robust. These data provide a source for future studies of novel SMC proteins related to pathological phenotype. For example, Numb was significantly decreased in SMC derived from patients with advanced cardiovascular disease. It is an inhibitor of Notch signaling originally described in Drosophila [36]. Numb is well characterized for its role in development, but a specific role in SMC has not been described. More recently, it has been considered for study as a potential therapeutic for prostate cancer as a counter to dysregulated Notch signaling that leads to hyperproliferation of tumor cells and increased angiogenesis [37]. It is interesting to consider how downregulation of this Notch inhibitor may impact disease state.

The successful derivation of SMC from carotid endarterectomy samples also provided a unique platform to study Notch signaling activity in SMC from diseased vessels. Although there has been little study of the specific roles of Notch2 or Notch3 in atherosclerosis, both proteins have major roles in regulating SMC phenotype. In our previous work using normal primary cultures of human SMC, we found that Jagged1 promoted a differentiated, contractile SMC phenotype. Interestingly, although signaling via both Notch 2 and Notch 3 promoted contractile protein production, selective signaling via Notch2 was required for Jagged1-mediated suppression of proliferation [9]. Given the interest in targeting the Notch pathway in cardiovascular diseases [38, 39], we addressed the impact of Jagged1-Fc 
stimulation on diseased SMC. Our experiments in this area support two conclusions: first, that proliferation of endarterectomy-derived SMC in culture is still suppressed by Jagged1/Notch2 signaling, and second, that Jagged1-Fc does not promote the contractile phenotype of SMC from diseased vessels, unlike its activity in normal SMC [9]. Thus, the considerations for targeting the Notch pathway in atherosclerosis are complex. Plaques with high SMC content have traditionally been considered more stable than plaques with low SMC content, but this conclusion is based on plaque smooth muscle content that is determined by contractile protein levels. This might lead to the hypothesis that encouraging SMC proliferation would be beneficial to plaque stability, but only if those SMC adopted a fully differentiated phenotype. Recently, it has been shown that plaque destabilizing cell types, such as macrophage [40] and chondrocytes [41], sometimes originate as SMC, but undergo a dedifferentiation process [5]. Without the ability to push SMC toward the contractile phenotype, treatments that would promote SMC proliferation could add both stabilizing and destabilizing cell types to lesions.

Another consideration is that various cell types within the atherosclerotic lesion may respond differently to Notch inhibition, depending on the type of inhibitor. For example, general inhibitors of Notch proteolytic processing (gamma secretase inhibitor) or transcriptional activation (dominant negative Mastermind-like 1), specific blocking antibodies to Notch ligands or Notch proteins, and decoys (soluble ligands) [42] may have diverse effects based on the cell types in which Notch signaling is blocked. Specific molecular targeting of a ligand or receptor has proven effective in some cases. In mouse atherosclerosis models, inhibition of the Notch ligand DLL4 with a neutralizing antibody suppressed atherogenesis concomitant with decreased macrophage infiltration into the plaque and decreased calcification [12, 13]. These studies indicate that Notch inhibition may be effective in treatment of atherosclerosis to control inflammation, but the effects of Notch inhibition on plaque SMC should also be considered when evaluating effectiveness of such treatments.

Acknowledgements This study was supported by NIH grant R01HL070865 to LL. Support for a research coordinator and procurement of human tissue was provided via a pilot project award from the Cardiovascular Research Institute at Maine Medical Center. Individuals who have greatly facilitated this project include MMC vascular surgeons Christopher Healey, Robert Hawkins, Elizabeth Blazick, and Paul Bloch, research coordinators Melissa Garrett and Dana Tripp, research assistant Debra Wright, and operating room nurse manager Cynthia Jones (all at Maine Medical Center). We thank Lauren Richey DVM, PhD (Tufts University) for assistance in histopathology of atherosclerotic plaques. Core facilities that assisted with tissue processing and histology (Grazina Mangoba and Mayasah Al Hashimi of the Histopathology and Histomorphometry Core) and mass spectrometry (Proteomics and Lipidomics Core Facility) were funded via NIH COBRE awards 8P30GM103392 (D. St. Germain, PI) and
1P20GM121301 (LL, PI). Partial support for the core facilities used in this research was provided by NIH grant U54GM115516 (C. Rosen, PI). JD-K was supported by fellowship 16PRE29870001 from the American Heart Association.

\section{Compliance with ethical standards}

Conflict of interest The authors declare that they have no conflict of interest.

\section{References}

1. Benjamin EJ, Blaha MJ, Chiuve SE, et al. Heart disease and stroke statistics-2017 update: a report from the American Heart Association. Circulation. 2017;135:e146-603.

2. Brown RA, Shantsila E, Varma C, et al. Current understanding of atherogenesis. Am J Med. 2017;130:268-82.

3. Ross R, Agius L. The process of atherogenesis--cellular and molecular interaction: from experimental animal models to humans. Diabetologia. 1992;35:S34-40.

4. Gomez D, Owens GK. Smooth muscle cell phenotypic switching in atherosclerosis. Cardiovasc Res. 2012;95:156-64.

5. Shankman LS, Gomez D, Cherepanova OA, et al. KLF4dependent phenotypic modulation of smooth muscle cells has a key role in atherosclerotic plaque pathogenesis. Nat Med. $2015 ; 21: 628-37$.

6. Caolo V, Schulten HM, Zhuang ZW, et al. Soluble Jagged-1 inhibits neointima formation by attenuating Notch-Herp2 signaling. Arterioscler Thromb Vasc Biol. 2011;31:1059-65.

7. Li Y, Takeshita K, Liu PY, et al. Smooth muscle Notch1 mediates neointimal formation after vascular injury. Circulation. 2009;119:2686-92.

8. Sakata Y, Xiang F, Chen Z, et al. Transcription factor CHF1/Hey2 regulates neointimal formation in vivo and vascular smooth muscle proliferation and migration in vitro. Arterioscler Thromb Vasc Biol. 2004;24:2069-74.

9. Boucher JM, Harrington A, Rostama B, et al. A receptor-specific function for Notch2 in mediating vascular smooth muscle cell growth arrest through cyclin-dependent kinase inhibitor 1B. Circ Res. 2013;113:975-85.

10. Fung E, Tang SM, Canner JP, et al. Delta-like 4 induces notch signaling in macrophages: implications for inflammation. Circulation. 2007;115:2948-56.

11. Nakano T, Fukuda D, Koga J, et al. Delta-like ligand 4-Notch signaling in macrophage activation. Arterioscler Thromb Vasc Biol. 2016;36:2038-47.

12. Fukuda D, Aikawa E, Swirski FK, et al. Notch ligand delta-like 4 blockade attenuates atherosclerosis and metabolic disorders. Proc Natl Acad Sci USA. 2012;109:E1868-77.

13. Fukuda D, Aikawa M. Expanding role of delta-like 4 mediated notch signaling in cardiovascular and metabolic diseases. Circ J. 2013;77:2462-8.

14. Briot A, Civelek M, Seki A, et al. Endothelial NOTCH1 is suppressed by circulating lipids and antagonizes inflammation during atherosclerosis. J Exp Med. 2015;212:2147-63.

15. Rizzo P, Mele D, Caliceti C, et al. The role of Notch in the cardiovascular system: potential adverse effects of investigational notch inhibitors. Front Oncol. 2014;4:384.

16. Aquila G, Pannella M, Morelli MB, et al. The role of Notch pathway in cardiovascular diseases. Glob Cardiol Sci Pract. 2013;2013:364-71.

17. Gillet LC, Navarro P, Tate $\mathrm{S}$, et al. Targeted data extraction of the MS/MS spectra generated by data-independent acquisition: a new 
concept for consistent and accurate proteome analysis. Mol Cell Proteom. 2012;11:O111 016717.

18. Mi H, Muruganujan A, Casagrande JT, et al. Large-scale gene function analysis with the PANTHER classification system. Nat Protoc. 2013;8:1551-66.

19. Miano JM. Vascular smooth muscle cell differentiation-2010. J Biomed Res. 2010;24:169-80.

20. Tang Y, Boucher JM, Liaw L. Histone deacetylase activity selectively regulates Notch-mediated smooth muscle differentiation in human vascular cells. J Am Heart Association. 2012; https://doi.org/10.1161/JAHA.112.000901.

21. Tang Y, Urs S, Boucher J, et al. Notch and transforming growth factor-beta (TGFbeta) signaling pathways cooperatively regulate vascular smooth muscle cell differentiation. J Biol Chem. 2010;285:17556-63.

22. Nus M, Martinez-Poveda B, MacGrogan D, et al. Endothelial Jag1-RBPJ signalling promotes inflammatory leucocyte recruitment and atherosclerosis. Cardiovasc Res. 2016;112:568-580.

23. Gamrekelashvili J, Limbourg FP. Rules of attraction - endothelial Notch signaling controls leukocyte homing in atherosclerosis via Vcam1. Cardiovasc Res. 2016;112:527-529.

24. Quillard T, Devalliere J, Coupel S, et al. Inflammation dysregulates Notch signaling in endothelial cells: implication of Notch2 and Notch4 to endothelial dysfunction. Biochem Pharmacol. 2010;80:2032-41.

25. Zhang Q, Wang C, Liu Z, et al. Notch signal suppresses Toll-like receptor-triggered inflammatory responses in macrophages by inhibiting extracellular signal-regulated kinase 1/2-mediated nuclear factor kappaB activation. J Biol Chem. 2012;287:6208-17.

26. Gonzalez MJ, Ruiz-Garcia A, Monsalve EM, et al. DLK1 is a novel inflammatory inhibitor which interferes with NOTCH1 signaling in TLR-activated murine macrophages. Eur J Immunol. 2015;45:2615-27.

27. Kimball AS, Joshi AD, Boniakowski AE, et al. Notch regulates macrophage-mediated inflammation in diabetic wound healing. Front Immunol. 2017;8:635.

28. Sweeney C, Morrow D, Birney YA, et al. Notch 1 and 3 receptor signaling modulates vascular smooth muscle cell growth, apoptosis, and migration via a CBF-1/RBP-Jk dependent pathway. Faseb J. 2004;18:1421-3.
29. Morrow D, Guha S, Sweeney C, et al. Notch and vascular smooth muscle cell phenotype. Circ Res. 2008;103:1370-82.

30. Pessi T, Viiri LE, Raitoharju E, et al. Interleukin-6 and microRNA profiles induced by oral bacteria in human atheroma derived and healthy smooth muscle cells. Springerplus. 2015; 4:206.

31. Aird WC. Endothelial cell heterogeneity. Cold Spring Harb Perspect Med. 2012;2:a006429.

32. Regan ER, Aird WC. Dynamical systems approach to endothelial heterogeneity. Circ Res. 2012;111:110-30.

33. Yuan L, Chan GC, Beeler D, et al. A role of stochastic phenotype switching in generating mosaic endothelial cell heterogeneity. Nat Commun. 2016;7:10160.

34. Coen M, Marchetti G, Palagi PM, et al. Calmodulin expression distinguishes the smooth muscle cell population of human carotid plaque. Am J Pathol. 2013;183:996-1009.

35. Langley SR, Willeit K, Didangelos A, et al. Extracellular matrix proteomics identifies molecular signature of symptomatic carotid plaques. J Clin Invest. 2017;127:1546-60.

36. Frise E, Knoblich JA, Younger-Shepherd S, et al. The Drosophila Numb protein inhibits signaling of the Notch receptor during cellcell interaction in sensory organ lineage. Proc Natl Acad Sci USA. 1996;93:11925-32.

37. Flores AN, McDermott N, Meunier A, et al. NUMB inhibition of $\mathrm{NOTCH}$ signalling as a therapeutic target in prostate cancer. Nat Rev Urol. 2014;11:499-507.

38. Andersson ER, Lendahl U. Therapeutic modulation of Notch signalling--are we there yet? Nat Rev Drug Discov. 2014;13: $357-78$.

39. Redmond EM, Guha S, Walls D, et al. Investigational Notch and Hedgehog inhibitors--therapies for cardiovascular disease. Expert Opin Investig Drugs. 2011;20:1649-64.

40. Feil S, Fehrenbacher B, Lukowski R, et al. Transdifferentiation of vascular smooth muscle cells to macrophage-like cells during atherogenesis. Circ Res. 2014;115:662-7.

41. Naik V, Leaf EM, Hu JH, et al. Sources of cells that contribute to atherosclerotic intimal calcification: an in vivo genetic fate mapping study. Cardiovasc Res. 2012;94:545-54.

42. Dikic I, Schmidt MH. Notch: implications of endogenous inhibitors for therapy. Bioessays. 2010;32:481-7. 